\title{
Pädagogik zu Antifeminismus
}

\section{Bedarfe - Vorgehen - Schlussfolgerungen}

\author{
UlLA WitTENZELLNER \& SARAH KLEMM
}

\section{ANTIFEMINISMUS - WAS VERSTEHEN WIR DARUNTER?}

In den letzten Jahren hat sich auf dem Weg zu mehr Geschlechtergerechtigkeit in Deutschland einiges getan: die Öffnung der Ehe für gleichgeschlechtliche Paare, die Einführung des dritten Geschlechtseintrags „divers“, die Tatsache, dass Transgeschlechtlichkeit in der ICD-11 (International Classification of Deseases) der Weltgesundheitsorganisation nicht länger als psychische Krankheit gilt, das neue Sexualstrafrecht mit dem Grundsatz „Nein heißt Nein“. Langjährige Kämpfe von Frauen, lesbischen, schwulen, bisexuellen, trans*, inter* und queeren (lsbtiq+) Personen und Verbündeten haben für Fortschritte in Politik und Gesetzgebung gesorgt.

Gleichzeitig stehen diese Errungenschaften unter enormem Druck: Religiösfundamentalistische, ultra-konservative, rechtsextreme, rechtspopulistische und völkisch-nationale Akteur_innen ${ }^{1}$ agieren vehement gegen Geschlechtergerechtigkeit und vielfältige Lebens-, Liebens- und Familienentwürfe. So wird das Recht auf Schwangerschaftsabbruch in Frage gestellt, sexuelle und geschlechtliche Vielfalt wird als ,unnatürlich“ und die Vermittlung vielfältiger Lebensweisen in Schule und Kita unter dem Schlagwort ,Frühsexualisierung ‘ als Gefährdung von Kindern verunglimpft. Geschlechterforschung wird als Ideologie diffamiert und Personen, die sich feministisch und für vielfältige Lebens- und Liebensweisen positionieren, werden angegriffen und bedroht. „Überall geht es darum, einer real gelebten Vielfalt geschlechtlicher, sexueller und familialer Lebensweisen eine autoritäre Einfalt entgegenzusetzen, das Individuum in eine vorgegebene, traditionelle Geschlechterschablone zu stecken“ (Lang 2019: 43).

1 Wir verwenden den Unterstrich, um in unserer Schreibweise sichtbar zu machen, dass es mehr als zwei Geschlechter gibt und dass wir alle Geschlechter meinen. 
Als Sammelbegriff und verbindendes Element dieser verschiedenen Positionen, Akteur_innen und Organisierungen, die sich gegen die Akzeptanz geschlechtlicher und sexueller Vielfalt sowie gegen Errungenschaften und Forderungen gleichstellungsorientierter und emanzipatorischer Bewegungen richten, verwenden wir den Begriff Antifeminismus. Antifeminismus ist u.a. ein zentraler Bestandteil von rechten und extrem rechten Weltbildern:

„Traditionellen Entwürfen von Geschlecht kommt in den Diskursen und Praxen der extremen Rechten eine gleichbleibend hohe Bedeutung zu. Das Konstrukt der ,Volksgemeinschaft‘ bildet mit seinen geschlechtsbezogenen Platzanweisern und dem traditionellen verengten Familienbild die ideologische Klammer dieses vermeintlich naturhaften Entwurfs. Innere wie äuBere Bedrohungen scheinen die vermeintlich natürliche Stabilität der, Volksgemeinschaft‘ ins Wanken zu bringen: Während die Flexibilisierung traditioneller Geschlechterrollen dabei als innerer Feind imaginiert wird, gilt u.a. Migration als Bedrohung von außen“ (Laumann 2014: 20).

Antifeministische Narrative gehen jedoch nicht nur von rechtsextremen, sondern auch von christlich-fundamentalistischen und maskulistischen Akteur_innen aus (Lang/Peters 2018a: 19f). Sie haben zudem eine breite Sog- und Strahlwirkung in weite Teile der Gesellschaft hinein.

Antifeministische Akteur_innen sind zunehmend erfolgreicher, nicht zuletzt durch die Nutzung digitaler Räume und Sozialer Medien. Die im Internet vorherrschende Diskussionskultur - emotionale Empörungswellen - bieten ihnen Raum, ihr Gedankengut zu verbreiten. Das Internet ist dabei nicht die Ursache für Hass und Diskriminierung, es wirkt als Instrument aber dynamisierend. Insbesondere für Jugendliche und junge Erwachsene sind Soziale Medien und Plattformen wie YouTube, Facebook, Instagram und TikTok zentrale Räume der Vernetzung und Informationsbeschaffung sowie der Sozialisation und Identitätsfindung - unter den heutigen 14- bis 24-Jährigen gibt es keine Offliner mehr (DIVSI 2018: 15). Durch die Raumgewinne antifeministischer und rechter Positionen im Netz sind junge Menschen insofern besonders gefährdet, Affinitäten zu rechten und extrem rechten Szenen zu entwickeln. Jugendliche und junge Erwachsene sind dementsprechend eine der wichtigsten Zielgruppen (extrem) rechter Akteur_innen im Internet (Jugendschutz.net 2017: 8). Dies ist einer der Gründe für die Notwendigkeit einer kritischen pädagogischen Auseinandersetzung mit dem Thema Antifeminismus. 


\title{
2. WAS hat PÄdAgogIK MIt ANTIFEMINISMUS ZU TUN?
}

Grundlegender Teil antifeministischer Deutungsangebote sind sexistische, homo-, trans*- und inter*feindliche Positionen. Diese werden jedoch nicht nur von antifeministischen Akteur_innen vertreten, sondern beruhen auf Machtverhältnissen und historisch gewachsenen diskriminierenden Strukturen. Die Gesellschaft, in der wir leben, ist beispielsweise heteronormativ strukturiert. Damit ist die Annahme gemeint, es sei ,normal', dass alle Menschen auf Grundlage biologisch definierter körperlicher Merkmale zweifelsfrei und lebenslang genau einem von zwei Geschlechtern angehören und das jeweils andere der beiden Geschlechter sexuell und romantisch begehren. Heteronormativität ist tief ins Bewusstsein, in Narrative und Institutionen eingeschrieben. Nicht jede Person, die diesen Annahmen folgt, ist gleich Antifeminist_in. Dennoch wirkt diese Struktur diskriminierend gegenüber allen, die nicht in Normvorstellungen von Zweigeschlechtlichkeit und/oder Heterosexualität passen, weil sie z.B. weder männlich noch weiblich sind, oder nicht heterosexuell begehren. Solche Strukturen zeigen sich auch in Bildungs- und Ausbildungsstätten, Einrichtungen der Jugendarbeit und -freizeitgestaltung, wo Ungleichheitsverhältnisse wie Sexismus, Homo-, Trans*- und Inter*feindlichkeit (sowie darüber hinaus Rassismus, Antisemitismus, Klassismus, Diskriminierung von Menschen mit Behinderungen und weitere) eine Rolle spielen. Hiermit ist nicht gemeint, dass zweigeschlechtlich und heteronormativ strukturierte Bildungseinrichtungen per se antifeministisch sind. Jedoch:

\begin{abstract}
„Abgeleitet von gesellschaftlichen Rahmenbedingungen wird auch im Bildungswesen nach dem Prinzip der Zweigeschlechtlichkeit gestaltet, gehandelt und gelehrt. Diese gesellschaftliche Vorgabe und die damit verbundenen Rollenvorstellungen und Rollenhierarchien haben einen entscheidenden Einfluss auf die Identitätsentwicklung von Schüler_innen und Konsequenzen im Sinne von Diskriminierungen und Ungleichheiten. “ (Antidiskriminierungsstelle des Bundes 2013: 21)
\end{abstract}

Laut einer Studie des Deutschen Jugendinstituts aus dem Jahr 2015 wurden mehr als 50 Prozent der befragten 1sbt ${ }^{2}$ Jugendlichen in Bildungs- und Ausbildungsstätten beschimpft, beleidigt oder lächerlich gemacht (Deutsches Jugendinstitut 2015: 22). In Schulbüchern, Unterrichtsmaterialien und -inhalten wird geschlechtliche und sexuelle Vielfalt nach wie vor häufig nicht mitgedacht, verbesondert und pathologisiert (Bittner 2011: 74ff). Aufgrund dessen fließt viel Energie von 1sbtiq+ Kindern

2 Inter* Jugendliche wurden in der Studie nicht adressiert. Die Befragung richtete sich an lesbische, schwule, bisexuelle und trans* Jugendliche. 
und Jugendlichen in den Umgang mit Diskriminierung. $\mathrm{Zu}$ den möglichen Folgen gehören Schuldistanz und -abbruch (Laumann/Debus 2018a: 6).

Diesen Ungleichheits- und Diskriminierungsverhältnissen entgegenzuwirken und allen Kindern, Jugendlichen und Erwachsenen gleichberechtigte Teilhabe an Bildungsprozessen $\mathrm{zu}$ ermöglichen, ist eine gesamtgesellschaftliche Herausforderung. Dazu gehört, eine inklusive Lernumgebung zu schaffen, die allen Menschen „persönliche Entwicklung und gesellschaftliche Teilhabe“ (Debus 2018: 87) ermöglicht. Das Grundgesetz und internationale menschenrechtliche Verträge stärken dabei all jenen Fachkräften und Einrichtungen den Rücken, die sich für den Abbau diskriminierender Strukturen einsetzen. Sie beinhalten rechtliche Vorgaben und Maßstäbe für den gesamten schulischen und außerschulischen Bildungsbereich, u.a. Diskriminierungs- und Gewaltfreiheit, sowie die Förderung des Bewusstseins für die Menschenrechte (Cremer 2019: 12). So muss Bildung laut UN-Sozialpakt beispielsweise ,auf die volle Entfaltung der menschlichen Persönlichkeit und des Bewusstseins ihrer Würde gerichtet sein und die Achtung vor den Menschenrechten und Grundfreiheiten stärken“ (Internationaler Pakt über wirtschaftliche, soziale und kulturelle Rechte vom 19. Dezember 1966, Artikel 13).

Diese ohnehin große Aufgabe wird jedoch durch antifeministische Mobilisierungen und deren Druck auf den Bildungsbereich erschwert: Antifeministische Positionen und Deutungsangebote stellen sich explizit gegen den Abbau von Diskriminierungsstrukturen, gegen sexuelle und geschlechtliche Vielfalt, Gleichberechtigung und vielfältige Lebensweisen. Sie stehen damit einer inklusiven Pädagogik auf Basis der Grund- und Menschenrechte entgegen. Verschiedene pädagogische Ansätze, insbesondere Sexualpädagogik und Pädagogik zu geschlechtlicher und sexueller Vielfalt, gegen die Diskriminierung von lesbischen, schwulen, bisexuellen, trans*, inter* und queeren (lsbtiq+) Personen, werden von antifeministischen Akteur_innen mit zunehmender Aggressivität angegriffen (Laumann/Debus 2018b: 275ff). Pädagog_innen, die sich vielfaltsbejahend positionieren, selbst nicht-normative Geschlechterrollen, Lebens- und Liebensweisen vorleben oder sich für Gleichberechtigung einsetzen, werden diffamiert und in ihrer Arbeit behindert. Fachkräfte, von Kita- bis Berufsschulpädagog_innen, berichten von rechten und antifeministischen Angriffen. So erzählten einige Fachkräfte in unseren Fortbildungen von vielfaltsfeindlichen Anliegen und Forderungen von Eltern, die sich auf die sich selbst so bezeichnende Initiative ,Besorgte Eltern 'beziehen. Auf Portalen wie ,elternaktion.com 'werden von besagter Initiative Anleitungen zur Verfügung gestellt, wie Eltern gegen eine Pädagogik der Vielfalt vorgehen können. Manche Fachkräfte berichten uns in Fortbildungen, dass sie die klare Befürwortung einer Pädagogik, die sich für geschlechtliche und sexuelle Vielfalt einsetzt, und eine Positionierung als feministisch aus Sorge vor Angriffen vermeiden.

Pädagogische Einrichtungen und Fachkräfte haben also einerseits den Auftrag, diskriminierungsfreie Lernräume zu schaffen, andererseits stehen sie selbst unter 
enormem Druck durch antifeministische Angriffe. Dies bedeutet für uns, Fachkräfte und Institutionen weiterzubilden und zu stärken in ihrem Engagement für demokratische, vielfaltsbejahende Lehre und Lernumfelder. Hierfür braucht es (u.a. pädagogische) Räume, die Kindern, Jugendlichen und Erwachsenen eine Auseinandersetzung mit Themen wie Liebe, Sexualität, Geschlecht, Familie usw. ermöglichen eben jene Themenfelder, die von Antifeminist_innen strategisch besetzt werden und dabei eine diskriminierungskritische, vielfaltsbejahende Haltung stärken. Ebenso braucht es (pädagogische) Räume, um Austausch über antifeministische Angriffe zu ermöglichen, Bestärkung zu erfahren und Umgangsmöglichkeiten zu erproben.

\section{Wie KANN PÄDAgogische ARBEIT ZU ANTIFEMINISMUS AUSSEHEN?}

Um uns dem Thema Antifeminismus pädagogisch zu nähern, arbeiteten wir im Modellprojekt Social Media Interventions! - rechtsextremen Geschlechterpolitiken im Netz begegnen ${ }^{3}$ mit unterschiedlichen pädagogischen Formaten einerseits für Jugendliche und junge Erwachsene und andererseits für pädagogische Fachkräfte und Multiplikator_innen. Wie unsere Formate für Fachkräfte und Multiplikator_innen gestaltet sind, skizzieren wir im Folgenden. Auf Bildungsangebote mit Jugendlichen gehen wir hier auf Grund der Kürze des Beitrags nicht näher ein. Viele unserer Überlegungen lassen sich jedoch auf Angebote für Jugendliche übertragen. Zunächst gehen wir auf die Grundlagen pädagogischer Arbeit zu Vielfalt und Geschlecht ein, um danach die Herangehensweise des Trägers Dissens - Institut für Bildung und Forschung e.V. und unseres Modellprojekts zu erläutern. Schließlich werden Schlussfolgerungen für eine pädagogische Arbeit zu Antifeminismus aus unseren Erfahrungen gezogen.

\subsection{Vorüberlegungen zu geschlechter- und vielfaltsorientierter pädagogischer Arbeit als Prävention von Antifeminismus}

Vielfaltsorientierte und geschlechterreflektierte Pädagogik bilden die Grundlage unserer pädagogischen Arbeit. Was verstehen wir darunter?

3 Gefördert vom Bundesministerium für Familie, Senioren, Frauen und Jugend im Rahmen des Programms Demokratie leben!, Laufzeit September 2017 bis Dezember 2019. Dissens e.V. war zudem Praxispartner des Projekts REVERSE - Krise der Geschlechterverhältnisse? Anti-Feminismus als Krisenphänomen mit gesellschaftsspaltendem Potenzial (Laufzeit: Oktober 2017 bis Januar 2020), das ebenfalls vom BMBF im Rahmen der Förderlinie Zusammenhalt stärken in Zeiten von Krisen und Umbrüchen finanziert wurde. 
Geschlechterreflektierte Pädagogik versucht vor dem Hintergrund verengender gesellschaftlicher Geschlechteranforderungen und -rollen einen Rahmen zu schaffen, in dem Kinder und Jugendliche aller Geschlechter die Möglichkeit haben, etwas auszuprobieren und Erfahrungen zu machen, ohne Angst zu haben, dafür abgewertet zu werden. Dazu kann die Förderung, geschlechtsuntypischer' Interessen und Fähigkeiten gehören - allerdings ohne den Aufbau neuer Ge- und Verbote und bei gleichzeitiger Wertschätzung auch für ,typische' Interessen und Fähigkeiten. Geschlechterreflektierte pädagogische Ansätze zielen zudem darauf, alle Lernenden von Geschlechteranforderungen zu entlasten und bei Gewalt und Diskriminierung klare Grenzen zu setzen (vgl. hierzu Dissens e.V. \& Debus et al. 2012).

Pädagogik zu geschlechtlicher und sexueller Vielfalt ist als Teil inklusiver Pädagogik zu verstehen. Sie versucht vor dem Hintergrund der Diskriminierung von lesbischen, schwulen, bisexuellen, trans*, inter* und queeren Identitäten und Lebensweisen, die real existierende Vielfalt von sexuellen Orientierungen, Geschlechtsidentitäten und Ausdrucksweisen in pädagogischen Angeboten sichtbar zu machen und konsequent mitzudenken. Geschlechtliche, sexuelle und amouröse Vielfalt sollte dabei einerseits als Querschnittsthema berücksichtigt und andererseits gezielt thematisiert werden.

Vielfaltsorientierte Lernumgebungen und pädagogische Konzepte, die geschlechtliche und sexuelle Vielfalt von vornherein mitdenken, entlasten von Geschlechteranforderungen und wirken Diskriminierung und damit antifeministischen Einstellungen entgegen. Dies ermöglicht insbesondere lsbtiq + Kindern und Jugendlichen die Verwirklichung ihres Menschenrechts auf Bildung und eine gleichberechtigte Teilhabe an Lernprozessen sowie allen Formen des sozialen Austauschs und Zusammenlebens in ihren Umfeldern.

Aber auch heterosexuelle und cisgeschlechtliche ${ }^{4}$ Kinder und Jugendliche profitieren von einem solchen Lernumfeld. Alle Kinder und Jugendlichen stehen unter dem Druck, als ,richtiger‘ oder ,normaler' Junge, bzw. als ,richtiges‘ oder ,normales' Mädchen wahrgenommen zu werden, um nicht ausgegrenzt, abgewertet oder in anderer Form verbesondert und sanktioniert zu werden. Ein inklusives Lernumfeld, das vielfältige Geschlechterrollen, geschlechtliche Identitäten, sexuelle Orientierungen und weitere Dimensionen menschlicher Vielfalt anerkennt und wertschätzt, entlastet alle Lernenden (und Lehrenden) von Druck und Anforderungen. Es kommt insofern allen Kindern, Jugendlichen und Pädagog_innen zugute.

Auch die Fragen, Ängste und Unsicherheiten von Erwachsenen benötigen einen möglichst sicheren Rahmen für Auseinandersetzung, Reflexion und Haltungsarbeit.

4 Cisgeschlechtliche (kurz ,cis`) Personen identifizieren sich mit dem Geschlecht, das ihnen bei Geburt, in der Regel aufgrund der gesellschaftlichen Einordnung ihrer Genitalien, zugewiesen wurde. 
Nicht selten begegnet uns beispielsweise in Seminaren, in denen wir geschlechtliche und sexuelle Vielfalt thematisieren und/oder Zweigeschlechtlichkeit infrage stellen, die Sorge vor dem Verlust der eigenen Zugehörigkeitskategorien (Debus 2012: 178). Wenn es mehr als zwei Geschlechter gibt, heißt das, ich darf keine Frau mehr sein? Sollen mir Interessen und Verhaltensweisen, die ich mit meinem Geschlecht verknüpfe und die zentraler Bestandteil meiner Identität sind, abgesprochen werden? Diese Sorge kann unter anderem aus dem Missverständnis entstehen, eine Kritik an starren und begrenzenden Vorstellungen von Geschlecht und Geschlechterrollen ginge mit dem Versuch einher, neue Normen oder Verhaltensgebote aufzustellen. Dies ist nicht der Fall: Geschlechterreflektierte pädagogische Ansätze, ebenso wie zahlreiche politische und zivilgesellschaftliche Bemühungen um Geschlechtergerechtigkeit, zielen nicht auf die Abschaffung alter Regeln zugunsten neuer Ge- und Verbote, sondern auf die Sichtbarmachung vielfältiger Lebensweisen und eine Erweiterung der Möglichkeiten für alle Menschen. „Zu verlieren gibt es nur das Privileg, meine eigene ,Richtigkeit‘ dadurch zu bestätigen, dass ich andere als ,falsch“ markiere“ (Debus 2012: 178). Gleichzeitig sind dieses und weitere Missverständnisse keineswegs überraschend, sondern verweisen auf den Bedarf nach (u.a.) pädagogischen Angeboten, die eine Auseinandersetzung mit den genannten Themenkomplexen ermöglichen.

Generell bieten Wissensvermittlung und Austausch zu vielfältigen Lebens- und Liebensweisen also allen Menschen alternative, positive Deutungsangebote als Entlastung von normativen Geschlechteranforderungen. Pädagogische Angebote, die die Auseinandersetzung mit Fragen um Geschlecht, Sexualität, Liebe und Beziehung ermöglichen, sollten daher nicht erst als Reaktion auf (antifeministische) Angriffe angeboten werden.

Einen besonderen Bedarf nach Qualifizierung haben Pädagog_innen und Multiplikator_innen, die im Rahmen unserer Angebote für Fachkräfte einerseits genau wie andere Teilnehmende inhaltlich weitergebildet und gestärkt werden, andererseits aber auch dazu befähigt werden sollen, selbstständig mit ihren Zielgruppen zu Themen rund um Antifeminismus sowie generell Geschlecht, Familie, Beziehung etc. $\mathrm{zu}$ arbeiten.

\subsection{Zugangswege und Bildungsangebote}

Wie gestalten wir auf Grundlage der obigen Überlegungen unsere konkrete Fortbildungspraxis?

Die Fortbildungen und Workshops des Projekts Social Media Interventions! waren kurzzeitpädagogische Formate mit einem Umfang von meist ein bis zwei Tagen. Längere Formate (Seminarwochen oder -reihen) ermöglichen eine vertiefte und umfassendere Auseinandersetzung, wurden aufgrund der Kürze des Modellpro- 
jekts jedoch nicht angeboten. Unsere Fortbildungen und Workshops unterschieden sich in der Zahl der Teilnehmenden stark (von Kleingruppen mit acht Teilnehmer_innen bis zu großen Formaten mit bis zu 40 Teilnehmer_innen).

Die pädagogischen Angebote des Modellprojekts Social Media Interventions! beruhten auf den in langen Jahren pädagogischer Praxis und Forschung erarbeiteten Grundlagen des Trägers Dissens - Institut für Bildung und Forschung e.V. In all unseren Bildungsformaten für Erwachsene, auch über das Projekt Social Media Interventions! hinaus, sind folgende Elemente enthalten:

-Wissensvermittlung, unter anderem in Form von Inputs sowie Gruppenarbeiten.

- Haltungsarbeit: Hierbei reflektieren Teilnehmer_innen beispielsweise eigene Erfahrungen und Positionen und befassen sich so einerseits mit der Wirkmächtigkeit vergeschlechtlichter Sozialisationsprozesse sowie andererseits mit dem eigenen pädagogischen Handeln: Welche Verhaltensweisen wurden mir nahegelegt, wofür wurde ich gelobt, wofür sanktioniert? Inwiefern gebe ich die Anforderungen, mit denen ich konfrontiert war und/oder bin, an meine Zielgruppe weiter? Viele Teilnehmer_innen erleben ein Gefühl der Ermüdung im Umgang mit antifeministischen Angriffen. Haltungsarbeit kann hier auch bedeuten, die eigenen Anliegen und Ziele zu fokussieren, statt sich primär an negativen Erfahrungen abzuarbeiten.

- Austausch- und Vernetzungsangebote: Austauschmöglichkeiten sind für die Stärkung der Teilnehmenden und die Fokussierung eigener Anliegen von zentraler Bedeutung. Es geht aber auch um Vernetzung über die Veranstaltung hinaus.

- Bestärkende und empowernde Elemente und Erweiterung der Handlungsmöglichkeiten, beispielsweise durch die gemeinsame Erarbeitung und Erprobung von Strategien im Umgang mit antifeministischen Angriffen.

- Methodisch-didaktische Kompetenzen und Thematisierung von Arbeitsbedingungen: In Fortbildungen für Fachkräfte reflektieren wir Methoden gemeinsam im Hinblick auf ihre Potenziale und Risiken. Wir thematisieren didaktische Fragen sowie Rahmenbedingungen pädagogischer Arbeit. Insbesondere letzteres soll auch der Überforderung entgegenwirken, die für viele Fachkräfte aus der Beschäftigung in schlecht bezahlten oder anderweitig ressourcentechnisch schlecht ausgestatteten Bereichen resultieren kann.

Uns ist grundsätzlich eine Haltung gemeinsamen Lernens wichtig, bei der wir von den Teilnehmenden, die Teilnehmenden voneinander und von uns lernen. Hierzu sind Wohlwollen und Fehlerfreundlichkeit ebenso zentral, wie die Bereitschaft Verantwortung für das eigene Handeln sowie für das Miteinander und die Atmosphäre in der Fortbildung zu übernehmen.

Mit dieser Rahmung bieten wir auch Fortbildungen und Workshops zum Thema Antifeminismus an. Dabei kann Antifeminismus zum einen als Thema im Zentrum 
stehen und bearbeitet werden. In diesen Fortbildungen werden Teilnehmende für antifeministische und rechte Ansprachen und Mobilisierungsstrategien online und offline sensibilisiert, Räume für die kritische Beschäftigung mit diesen Positionen werden eröffnet und Betroffene gestärkt.

Uns begegnet in unseren Fortbildungen ein großer Bedarf, über konkrete Erfahrungen mit antifeministischen Angriffen sowie sexistischen, homo-, trans*- und inter*feindlichen Alltagssituationen zu sprechen. Das gilt für Personen, die im Netz aktiv sind, ebenso wie für Personen, die offline zu Themen rund um Geschlecht arbeiten oder aktiv sind. Antifeministische Angriffe richten sich insbesondere gegen Frauen, trans*, inter* und nicht-binäre Personen - auch ohne, dass sich diese explizit mit Themen wie Geschlecht, Feminismus etc. befassen. Nahezu alle unsere Teilnehmer_innen berichten davon, schon mit antifeministischen Angriffen in Berührung gekommen zu sein.

Hier braucht es Bestärkung und Empowerment, Vernetzung und Austausch. Unsere Fortbildungen boten hierfür einen Raum. Insbesondere pädagogische Fachkräfte fühlen sich bei diesen Themen häufig in ihren Einrichtungen nicht ernst genommen oder werden als Expert_innen für Fragen sexueller und geschlechtlicher Vielfalt adressiert und damit alleingelassen - auch wenn es um antifeministische Angriffe geht. Vernetzung und Austausch mit Gleichgesinnten ist deshalb besonders wichtig.

In unseren Seminaren äußern zudem viele Teilnehmer_innen großes Interesse an Informationen und Wissensübertrag zu antifeministischen Strukturen und historischen Bezügen, beispielsweise dem Organisationsgrad antifeministischer Bewegungen und deren Vernetzung untereinander (,Demo für alle', ,Bundesverband Lebensschutz', u.v.m.). Uns ist dabei wichtig, dass die kritische Auseinandersetzung mit antifeministischen Positionen sachlich erfolgt, ohne neutral gegenüber diskriminierenden und menschenverachtenden Einstellungen und Strukturen zu sein: „Bildung und insbesondere politische Bildung ist nicht in dem Sinne neutral, dass sie wertneutral wäre." (Cremer 2019: 21f)

Zum anderen ist eine Grundüberzeugung unserer Arbeit, dass Bildung und Pädagogik vielfältige Deutungsangebote zugänglich machen müssen. Hierfür sind die oben erläuterten Ansätze geschlechterreflektierter Pädagogik und Pädagogik zu geschlechtlicher und sexueller Vielfalt von zentraler Bedeutung, weshalb in unseren Fortbildungen neben der gezielten Auseinandersetzung mit Antifeminismus vielfaltsbejahende Ansätze besprochen und diskutiert wurden. Diese wirken im besten Sinne primärpräventiv ${ }^{5}$ gegen antifeministische, vielfaltsfeindliche, diskriminierende, rechtsextreme und rechtspopulistische Einstellungen und Verhaltensweisen.

5 Primärprävention setzt an, bevor Kinder, Jugendliche oder andere Zielgruppen sich rechten Szenen und Organisierungen zugewandt haben. Sie ist abzugrenzen von Sekun- 
Bei Fortbildungen für pädagogische Fachkräfte stand neben Reflexion, Wissensübertrag und Diskussion der Übertrag in die eigene Praxis im Fokus. Wie eingangs erwähnt, existiert Antifeminismus nicht unabhängig von diskriminierenden gesellschaftlichen Strukturen. Eine Auseinandersetzung mit Antifeminismus bezieht also auch diese Strukturen mit ein. Für unsere Arbeit mit Fachkräften bedeutet das die Vermittlung von Methoden, mit denen alle Geschlechter und sexuellen Orientierungen in Lernräumen und -inhalten berücksichtigt und sichtbar gemacht werden können.

\section{Schlussfolgerungen aus unserer Arbeit}

Aus den Bedarfen und Erfahrungen aus unserer pädagogischen Arbeit ergeben sich folgende Schlussfolgerungen:

1. Pädagogische Angebote, die sich explizit mit Antifeminismus befassen, ermöglichen eine kritische Auseinandersetzung und stärken Personen, die selbst mit antifeministischen Angriffen auf ihre Arbeit und/oder ihre Person konfrontiert waren oder sind. Pädagogische Angebote mit dem Themenschwerpunkt Antifeminismus sind also ein wichtiger Baustein im Bemühen um Gleichberechtigung und Vielfalt.

2. Inklusive pädagogische Angebote, die geschlechtliche und sexuelle Vielfalt mitdenken, ermöglichen 1sbtiq + Kindern und Jugendlichen den barrierefreien Zugang zu Bildung und entlasten alle Lernenden von dem Druck, als ,richtiger Junge“ oder ,richtiges Mädchen` zu gelten, um nicht abgewertet und ausgegrenzt zu werden. Geschlechterreflektierte Pädagogik und Pädagogik zu vielfältigen Lebens- und Liebensweisen leisten einen Beitrag zum Abbau diskriminierender gesellschaftlicher Strukturen und können zu mehr Gleichberechtigung und Entfaltungsmöglichkeiten für alle beitragen. Sexuelle und geschlechtliche Vielfalt müssen als Querschnittsthemen aber auch explizit in Bildungs- und pädagogischen Einrichtungen implementiert werden. Grundlegende Auseinandersetzungen mit geschlechterreflektierter Pädagogik müssen allen Pädagog_innen bereits in der Ausbildung ermöglicht werden.

därprävention - der Arbeit mit Personen, die sich in einem Hinwendungsprozess zu rechten Lebenswelten befinden, mit dem Ziel der Distanzierung - und Tertiärprävention - der ausstiegsorientierten Arbeit mit Personen, die fest in rechte Szenen und Lebenswelten eingebunden sind (Debus/Laumann 2014: 162). 
Mit diesen zwei Vorgehensweisen lässt sich eine Pädagogik gestalten, die gegen Antifeminismus und für eine gleichberechtigte Gesellschaft eintritt.

Bei all unseren Ansätzen bleibt zu beachten, dass Antifeminismus nicht hauptsächlich pädagogisch ,gelöst‘ und bearbeitet werden kann und sollte. Zum einen, weil unsere pädagogischen Angebote primär dazu dienen, die Teilnehmer_innen in ihrer persönlichen Entwicklung und ihren Lernprozessen zu begleiten. Zum anderen, weil der Abbau der Diskriminierungsverhältnisse, die Teil antifeministischer Narrative sind, strukturelle Veränderungen erfordert, die von den Adressat_innen pädagogischer Angebote allein nicht herbeigeführt werden können. Der Einsatz gegen Diskriminierung, für Gleichberechtigung und die Anerkennung von Vielfalt, ist eine gesamtgesellschaftliche Aufgabe!

\section{LITERATUR}

Antidiskriminierungsstelle des Bundes (Hg.) (2013): Diskriminierung im vorschulischen und schulischen Bereich. Eine sozial- und erziehungswissenschaftliche Bestandsaufnahme. [https:/www.antidiskriminierungsstelle.de/SharedDocs/ Downloads/DE/publikationen/Expertisen/Expertise_Diskriminierung_im_ vorschulischen_und_schulischen_Bereich.pdf?_blob=publicationFile \&v=4; abgerufen am 09.09.2019].

Bittner, Melanie (2011): Geschlechterkonstruktionen und die Darstellung von Lesben, Schwulen, Bisexuellen, Trans* und Inter* (LSBTI) in Schulbüchern. [https://www.gew.de/index.php?eID $=$ dumpFile $\& \mathrm{t}=\mathrm{f} \& \mathrm{f}=25113 \&$ token $=$ da9eb1b 770b9761031934497b6a9d0c5af5665c7\&sdownload=\&n=Schulbuchanalyse web.pdf; abgerufen am 05.12.2019].

Cremer, Hendrik (2019): Das Neutralitätsgebot in der Bildung. Neutral gegenüber rassistischen und rechtsextremen Positionen von Parteien? Deutsches Institut für Menschenrechte. [https://www.institut-fuer-menschenrechte.de/fileadmin/user upload/Publikationen/ANALYSE/Analyse_Das_Neutralitaetsgebot_in_der_Bil dung.pdf; abgerufen am 05.09.2019].

Debus, Katharina (2012): „Vom Gefühl, das eigene Geschlecht verboten zu bekommen - Häufige Missverständnisse in der Erwachsenenbildung zu Geschlecht“, in: Dissens e.V./Debus/Könnecke/Schwerma/Stuve, Geschlechterreflektierte Arbeit mit Jungen an der Schule, S. 175-188.

Debus, Katharina (2018): „Was heißt das für die Praxis? Konzeptionelle und didaktische Zugänge zu geschlechtlicher, amouröser und sexueller Vielfalt in der Pädagogik“, in: Debus/Laumann, Pädagogik geschlechtlicher, amouröser und sexueller Vielfalt - Zwischen Sensibilisierung und Empowerment, S. 87-120.

Debus, Katharina/Laumann, Vivien (2014): ,,Von der Suche nach männlicher Souveränität und natürlicher Weiblichkeit. Geschlechterreflektierte Rechtsextremis- 
musprävention unter den Vorzeichen von Geschlechteranforderungen und subjektiver Funktionalität“, in: Dies. (Hg.), Rechtsextremismus, Prävention und Geschlecht. Vielfalt_Macht_Pädagogik (= Reihe Arbeitspapiere der HansBöckler-Stiftung), Düsseldorf, S. 146-170.

Debus, Katharina/Laumann, Vivien (Hg.) (2018): Pädagogik geschlechtlicher, amouröser und sexueller Vielfalt - Zwischen Sensibilisierung und Empowerment, Berlin: Dissens - Institut für Bildung und Forschung e.V.

Deutsches Institut für Vertrauen und Sicherheit im Internet (DIVSI) (2018): Euphorie war gestern - Die ,Generation Internet` zwischen Glück und Abhängigkeit. [https://www.divsi.de/wp-content/uploads/2018/11/DIVSI-U25-Studie-eupho rie.pdf; abgerufen am 05.12.2019].

Deutsches Jugendinstitut (2015): Coming-out - und dann...?! [https://www.dji. de/fileadmin/user_upload/bibs2015/DJI_Broschuere_ComingOut.pdf; abgerufen am 10.09.2019].

Dissens e.V./Debus, Katharina/Könnecke, Bernard/Schwerma, Klaus/Stuve, Olaf (Hg.) (2012): Geschlechterreflektierte Arbeit mit Jungen an der Schule. Texte zu Pädagogik und Fortbildung rund um Jungen, Geschlecht und Bildung, Berlin: Dissens - Institut für Bildung und Forschung e.V.

Internationaler Pakt über wirtschaftliche, soziale und kulturelle Rechte vom 19. Dezember 1966. [https://www.sozialpakt.info/internationaler-pakt-ueber-wirtschaft liche-soziale-und-kulturelle-rechte-3111/; abgerufen am 05.09.2019].

Jugendschutz.net (2017): Vernetzter Hass - Wie Rechtsextreme im Social Web Jugendliche umwerben. [https:/www.jugendschutz.net/fileadmin/download/pdf/ Broschuere_Vernetzter_Hass.pdf; abgerufen am 06.08.2019].

Lang, Juliane (2019): „Geschlecht als Kampfarena“, in: Weiterdenken - Heinrich Böll Stiftung Sachsen: Politik im autoritären Sog. [http://www.weiterdenken.de/ sites/default/files/politik-im-autoritaren-sog-teil-1u2.pdf; abgerufen am 05.12. 2019].

Lang, Juliane/Peters, Ulrich (2018) (Hg.): Antifeminismus in Bewegung. Aktuelle Debatten um Geschlecht und sexuelle Vielfalt, Hamburg: Marta Press.

Lang, Juliane/Peters, Ulrich (2018a): „Antifeminismus in Deutschland. Einführung und Einordnung des Phänomens", in: Dies., Antifeminismus in Bewegung. Aktuelle Debatten um Geschlecht und sexuelle Vielfalt, S. 13-35.

Laumann, Vivien (2014): „(R)echte Geschlechter? - Die Bedeutung von Geschlecht für rechte Ideologien und Lebenswelten“, in: Katharina Debus/Vivien Laumann (Hg.), Rechtsextremismus, Prävention und Geschlecht, Berlin: Hans Böckler Stiftung, S. 19-30.

Laumann, Vivien/Debus, Katharina (2018a): „Einleitung“, in: Debus/Laumann, Pädagogik geschlechtlicher, amouröser und sexueller Vielfalt - Zwischen Sensibilisierung und Empowerment, S. 6-11.

Laumann, Vivien/Debus, Katharina (2018b): „,Frühsexualisierung “ und ,Umerziehung'? Pädagogisches Handeln in Zeiten antifeministischer Organisierungen 
und Stimmungsmache“, in: Lang/Peters, Antifeminismus in Bewegung. Aktuelle Debatten um Geschlecht und sexuelle Vielfalt, S. 275-302. 
